\title{
Natural vs Anthropogenic Risk Reduction: Facing Invasion Risks Involving Multi-Stable Outcomes
}

David Finnoff, Associate Professor, Department of Economics and Finance, University of Wyoming, finnoff@uwyo.edu

Richard D. Horan, Professor, Department of Agricultural, Food, and Resource Economics, Michigan State University, horan@msu.edu

Jason F. Shogren, Stroock Professor, Department of Economics and Finance, University of Wyoming, jramses@uwyo.edu

Carson Reeling, Research Assistant, Department of Agricultural, Food, and Resource Economics, Michigan State University, carson.reeling@gmail.com

Kevin Berry, Postdoctoral Associate, Yale School of Forestry and Environmental Studies, Yale University, kevin.berry@yale.edu

12 April 2016

Acknowledgements: We gratefully acknowledge funding from NSF grant \#1414374 as part of the joint NSF-NIH-USDA Ecology and Evolution of Infectious Diseases program, and by UK Biotechnology and Biological Sciences Research Council grant BB/M008894/1. We also gratefully acknowledge funding from NOAA CSCOR Grant No. NA09NOS4780192, the National Institute of General Medical Sciences (NIGMS) at the National Institutes of Health, grant \#1R01GM100471-01, and USDA National Institute of Food and Agriculture, Grant \#2011-67023-30872. The contents of the paper are solely the responsibility of the authors and do not necessarily represent the official views of NSF, NOAA CSCOR, NIGMS, or USDA. 


\begin{abstract}
Natural resources important to economic systems are managed in the presence of event uncertainty, marking the transition from a more desirable ex ante state to a less desirable ex post state. The risks of this transition are endogenous and depend on state of the natural resource system and human behavior. In designing least cost policies of risk reduction, policy makers should consider the optimal mix of natural and human risk reduction. Decisions about the best risk reduction mix matter most for systems vulnerable to ex post multi-stability, in which the natural resource system has the potential to begin in one of multiple, locally optimal basins of attraction. This is because ex ante decisions can affect the initial conditions for the ex post system, thereby determining the ex post basin of attraction and the optimal ex post state. In this paper, we find that an ex ante system that is convex and uniquely stable without risk may become non-convex and multi-stable in the presence of endogenous risks and ex post multistability. This result arises because ex post non-convexities, and the uncertainties associated with the invasion and its magnitude, can create ex ante non-convexities that generate multiple optimality candidates.
\end{abstract}




\section{Introduction}

Invasive species pose significant risks to biodiversity around the globe, by out-competing or preying upon native species and spreading diseases to wild and domestic animals and plants (MEA 2005). ${ }^{1}$ It has been well-established that these risks are endogenous (e.g., Shogren, 2000, Finnoff et al., 2013): humans can invest in self-protection or mitigation (lowering the chance of a loss) to influence the likelihood of invasion (e.g., Horan et al. 2002), in self-insurance or adaptation (lowering the realized loss) to affect the economic and ecological impacts after an invasion occurs (Knowler and Barbier 2000; Horan et al. 2011; Olson and Roy 2002; Perrings 2005; Fenichel et al. 2010), or in alternatives that provide both self-protection and self-insurance (lowering both the chance of a loss and the realized loss (e.g. Leung et al. 2002; Ehrlich and Becker,1972). Investments in natural risk reduction can involve enhancing the native system's ability to protect itself (through ecosystem services) or they can involve human interventions that directly protect the native system (biosecurity or trade measures to prevent invasion; removal or sterilization of invaders as a form of adaptation). Decisions about ex ante natural and anthropogenic risk reduction matter for systems vulnerable to ex post multi-stability, in which the invaded system can begin in one of multiple, locally optimal basins of attraction (e.g., the ecosystem remains productive or collapses). ${ }^{2}$ This is because ex ante decisions affect the initial conditions for the invaded system, which then determine the ex post basin of attraction and the optimal ex post management (Dasgupta and Maler 2003; Brock and Starrett 2003).

In theory, robust ecosystems can often protect themselves against invasion risks (e.g.,

\footnotetext{
${ }^{1}$ Invasive species pose other risks not directly related to biodiversity. For instance, their spread of diseases can ultimately reach humans (Daszak et al. 2000). They also damage or degrade assets such as power plants, boats, piers, and reservoirs (Perrings et al. 2000).

${ }^{2}$ Our use of the term multi-stability refers to bioeconomic multi-stability, for which the optimal strategy depends on the initial conditions. Moreover, environmental perturbations or shocks within multi-stable systems can have nonmonotonic effects by pushing the system into another basin of attraction, at which point the new initial conditions determine a newly optimal strategy (Crocker and Forester 1981; Brown et. al. 2011). Bioeconomic multi-stability generally stems from ecological or economic non-convexities.
} 
Crocker and Shogren, 2001). This ability has been called many things in the literature, such as resilience, persistence, and resistance (see Crocker, 1995). Here, we do not differentiate among the processes, and we define natural-insurance-cum-protection (NICP) as the ability of the natural ecosystem to protect itself, by both lowering the chance the ecosystem transitions to being invaded and lowering the expected losses that occur if the ecosystem becomes invaded. ${ }^{3}$ Environmental managers may invest in NICP by manipulating human activities that disturb the ecosystem (harvests, grazing) and allow the ecosystem to naturally combat the invader at any time during the invasion process. This investment in NICP is an investment in the ability of the ecosystem to protect itself. One example is rangeland. Relatively undisturbed perennialdominated rangelands can naturally resist invasions by annuals such as cheat grass, yet if the range is overused by humans (e.g., for grazing), the invading annuals can establish, spread and dominate. ${ }^{4}$ Similarly, more pristine fish populations in freshwater lakes prevent or adapt to rusty crayfish invasions (Drury and Lodge 2009). In these cases, investments involve foregoing more intensive human use of the resource.

Some ecosystems may be protected via direct anthropogenic interventions that prevent the introduction or establishment of the invaders without manipulating the natural system. Such interventions can be particularly effective when the native system in its current state represents a prime ecological niche for the invader. Interventions include self-protection (SP) efforts that lower the chance the invader can establish (e.g., through early response and eradication programs, and restrictions on activities that allow long-distance dispersal) or self-insurance (SI) efforts that promote adaptation and loss reduction if the invasion occurs (e.g., direct removals or

\footnotetext{
${ }^{3}$ We are following Lee's (1998) definition-self-insurance-cum-protection (SICP), which implies investments in risk reduction affect both the likelihood of realizing a bad state of nature (self-protection) and the severity of the bad state (self-insurance).

${ }^{4}$ Over-used rangeland systems are particularly vulnerable to wildfire, which facilitates long-term changes in vegetation (Kobayashi et al. 2014; Perrings and Walker 1997).
} 
sterilization of the invader).

Whether invasive species risks can be reduced by investments in natural or anthropogenic risk reduction, or both, depends on the state of the ecosystem. The timing of the investments in relation to the invasion event plays an important role. Traditionally the economics literature on invasive species has focused on either an ex ante or ex post approach, not both. But treating ex ante and ex post methods as separable implies ex post invasion risks are treated as exogenous ex ante, such that the potential economic and ecological consequences of an invasion are unaffected by ex ante decisions. Such a perspective is naïve (Keller 2009). For example, aquatic invasions are often predictable events (Kolar and Lodge 2001, 2002) influenced by human activities (Herborg et al. 2009; Perrings et al. 2000) such as trade flows that facilitate long distance dispersal of invasive species (Bossenbroek, et al. 2001; Prasad et al. 2010). Moreover decisions about natural and anthropogenic risk reduction affect propagule pressure (e.g, Kolar and Lodge 2001, 2002; Herborg et al. 2009; Perrings et al. 2000) as well as ecological interactions that determine the initial prevalence of the invader and whether the system can protect itself in the invaded state. ${ }^{5}$ An endogenous risk approach (Ehrlich and Becker,1972; Shogren and Crocker, 1991) takes these impacts into account so that managers need to consider both ex ante and ex post insurance decisions simultaneously when thinking about ex ante risk reduction.

Prior work on the ex ante management of systems facing invasion risks has neither addressed ex post multi-stability nor included the other key features of our model: the likelihood of an invasion and its magnitude being conditional on ex ante investments in natural insurance

\footnotetext{
${ }^{5}$ The state of the non-invaded system may play an important role for invasion success (Case 1990; Namba and Takahashi 1993; Davis et al. 2000; Gilligan and van den Bosch 2008; Drury and Lodge 2009). Thriving native competitor or predator populations may prevent entry by potential invaders, whereas depleted native populations may create room for entry. The opposite is true for native prey species, which would include susceptible hosts for pathogens.
} 
(i.e, the state of the non-invaded system). ${ }^{6}$ Only Olson and Roy (2005) and Kim et al. (2006) model invasion likelihood and magnitude, but they only consider the effects of anthropogenic insurance. Ranjan et al. (2003) and Horan and Fenichel (2007) model the likelihood of invasion to depend on ex ante investments in natural insurance, but both analyses treat the invasion magnitude as exogenous.

Herein we capture this linkage between the ex post outcomes and the ex ante invasive species risk management problem given natural and anthropogenic risk reduction. The key result that emerges from our dynamic endogenous risk model is that an ex ante system that is convex and uniquely stable without invasion risk may become non-convex and multi-stable in the presence of endogenous invasion risks and ex post multi-stability. This result arises because the ability of the natural population in providing NICP, in terms of effects on ex post nonconvexities, on managing invasion hazards in conjunction with anthropogenic risk reduction, and on the expected economic impacts of an invasion, can create ex ante non-convexities that generate multiple optimality candidates.

\section{The Model}

Consider a managed, native ecological system that is measured by the state variable $N(t)$ (e.g., wildlife abundance or an index of the condition of a rangeland), where management might involve livestock grazing or harvests. This state is at risk from the potential introduction of an invasive species. We assume for simplicity that introduction and establishment of the invader, and the economic realization of these processes, occur simultaneously to produce an invasion at some date $T$. This allows us to consider two time intervals: the ex ante or pre-invasion interval $t$

\footnotetext{
${ }^{6}$ Analyses of ex ante management in the invasive species context are less common than those of ex post management (e.g., Knowler and Barbier 2000; Horan et al. 2011; Olson and Roy 2002; Perrings 2005; Fenichel et al. 2010; Fenichel and Horan 2007).
} 
$<T$, and the ex post or post-invasion interval $t \geq T$. Most of our analysis focuses on the ex ante management of $N(t)$. But since managers need to consider the potential ex post impacts of ex ante decisions, we begin by briefly characterizing the basic economic features of the ex post system.

\section{The expected ex post economic outcome}

Given our ex ante focus, we do not focus on the specific economic and ecological relations associated with ex post management. Rather, we rely on the findings of previous research, which demonstrated the potential for multiple stable bioeconomic equilibria, or multi-stability, in invaded systems. Prominent examples include changes in the composition of vegetation on semi-arid rangeland following anthropogenic or natural perturbations (Perrings and Walker 1997; Finnoff, Strong and Tschirhart 2008; Kobayashi et al. 2014), potential for foodweb perturbations following aquatic invasions (Horan et al., 2011), and in the management of an invader spreading across a system of lakes (Finnoff et al., 2010).

This prior work has a common theme in that invaded systems are characterized by a relatively "good" equilibrium, with relatively small abundances of the invader and/or little damage, and a "bad" equilibrium, with high invader abundances and significant damage. The "good" equilibria typically have higher levels of welfare than the "bad", although costs and benefits along the transition to an equilibrium also matter. Taking into account the net benefits of transitioning to an equilibrium, there may be a threshold set of initial conditions, or a "Skiba" threshold, along which managers are indifferent between pursuing either long-run outcome. This threshold divides the state space into two basins of attraction: one in which the good equilibrium is optimally pursued, and another in which the bad equilibrium is optimal. 
For simplicity, the ex post setting is modeled by the present value of net benefits for the post-invasion interval. These net benefits are developed formally in Appendix A1; we only provide an overview here. Suppose an invasion at time $t=T$ produces an initial level of invasion pressure, denoted $I$. The value of $I$ is random, with smaller values more likely when the managed stock is larger. Regardless of the level of pressure, the dynamic system exhibits bioeconomic multi-stability with two basins of attraction indexed by $i$ : a good basin $(i=g)$ and a bad basin $(i=b) .^{7}$ The net benefits in each basin $i$, denoted $v^{i}$ are assumed to be known increasing and concave function of $N(T)$. We assume $v_{I}^{i}<0$ when $N$ is sufficiently small, as the invader adversely impacts the native stock in this case (e.g., via inter-species competition or predation). We allow for the possibility that $v_{I}^{i}>0$ when $N$ is large, as the invader might serve as prey for the native stock in that case. ${ }^{8}$

The combination of $I$ and $N(T)$ determines whether the invaded system alights in the good or bad basin of attraction. Specifically, for a given $N(T)$, the system will be in the good basin when $I<I^{*}(N)$, where $I^{*}(N)$ represents the Skiba threshold that separates the basin. We assume $I^{* \prime}(N)>0$ so that the good basin of attraction is expanded for larger values of $N$. This means a larger value of $N(T)$ is more likely to place the system in the good basin of attraction, thereby providing natural protection against invasion losses. ${ }^{9}$ Since $N(T)$ is determined ex ante, this

\footnotetext{
${ }^{7}$ Our assumption that two basins of attraction arise for each value of $I$ is made to simplify the mathematical analysis in the Appendix. Specifically, this assumption allows our expected ex post value function $W(N)$, including the Skiba threshold affecting this function, to be a continuously differentiable function of $N$. The assumption of two stable equilibria, rather than more, is also made for simplicity: two stable equilibria are all that is required to illustrate how ex post multi-stability can lead to ex ante multi-stability.

${ }^{8}$ For instance, invasive rusty crayfish generate a net adverse impact on native bass populations when bass populations are small, but can serve as a net source of prey when the bass populations are large (Horan et al. 2011). In the Great Lakes, invasive Round Gobys are now an important prey resource to highly valued sport fish (http://www.lakescientist.com/ecological-impacts-invasive-round-goby-neogobius-melanostomus-laurentian-greatlakes-beyond-summary-presentations-iaglr-2014/). ${ }^{8} I$ may also provide direct benefits or costs, such as changes in amenity values of other directly-valued services (e.g., zebra mussels can improve local water clarity in addition to negatively impacting foodwebs). However, we assume the ecological values dominate.

${ }^{9}$ A larger $N$ may not provide self-insurance in all settings. For instance, infectious diseases generally have an easier time invading a dense population. This case is examined in Horan et al. (2016).
} 
protection may be viewed ex ante as a form of natural insurance against potential ex post losses.

Ex ante, uncertainty exists about magnitude of the initial invasion pressure $I$ that will arise at time $T$. Invasion pressure is treated as a random variable whose pdf depends on $N(t)$, with a more pristine system (a larger $N(T)$ ) reducing the likely value of $I$ in the sense of firstorder stochastic dominance. This is the case when the invader competes with natives for resources or serves as a prey for the natives, such that a more pristine setting prevents the native system from being dominated by the invader (e.g., a relatively pristine rangeland system with perennial grasses prevents the invaded system from switching to primarily invasive annual grasses).

From an ex ante perspective, and dropping the time indices for simplicity, the expected present value of ex post net benefits are denoted $W(N)$, which reflects both the uncertain level of invasion pressure and ex post multi-stability. We assume $W(0) \leq 0$, and it is easily verified that $W_{N}(N)>0$ (see Appendix A1). The following proposition addresses the second derivative:

Proposition 1. W(N) is concave in the absence of ex post multi-stability or ex ante uncertainty about $I$. However, either ex post multi-stability or ex ante uncertainty about $I$ (or both) may cause $W(N)$ to be convex over a range of $N$.

Proof: See the Appendix A1.

\section{The ex ante economic problem}

Now consider the ex ante problem. The native ecological state is controlled by the variable $h$ to provide flows of benefits and costs, where $h$ could represent harvests, silviculture, or grazing.

Each unit of $h$ generates a net unit value of $B(N)$, which is an increasing and concave ( $B_{N}>0$, 
$\left.B_{N N}<0\right)$ with $B(0)<0, B\left(N^{\pi=0}\right)=0$, and $B(N)>0 \forall N>N^{\pi=0}$, where $N^{\pi=0}$ is the zero profit threshold. The control $h$ may act as a source of mortality on the managed state (for the case of harvests or grazing) so the pre-invasion equation of motion is

(1) $\quad \dot{N}=g(N)-h$

where $g$ represents net growth of managed state $N$. We assume $g$ is concave with $g(0)=g(K)=$ 0 , where $K$ is the natural equilibrium prior to invasion. Also, for a given discount rate $r$, assume $g_{N}(0)>r$ to ensure extinction is sub-optimal.

For our purpose, reductions in $h$ can be viewed as an investment in natural insurancecum-protection (NICP), in that a sufficiently small $h$ leads to an increase in $N$ and hence the overall level of NICP. NICP involves three components. The first two components have already been described: a larger $N$ reduces the likely initial invasion pressure $I$, and it also increases the size of the good basin of attraction. The third component is that a larger $N$ reduces the probability of invasion. We treat uncertainty about introduction and establishment as uncertainty regarding the invasion date $T$ (e.g., Reed and Heras 1992). Let the probability an invasion occurs at any time $t$, conditional on it not already having occurred, be given by the hazard rate $\psi(N, z)=\lim _{\Delta t \rightarrow 0}\left\{\frac{\operatorname{Pr}(\text { Invasion in }(t, t+\Delta t)) \mid \text { no invasion }}{\Delta t}\right\}$, where control variable $z$ is anthropogenic self-protection (SP) that reduces invasion hazards, $\psi_{z}(N, z)<0$. We assume $\psi_{z z}(N, z)>0$, with $\psi_{z}(N, 0)=-\infty$ and $\psi_{z}(N, \infty)=0$, so that there are diminishing returns to SP in reducing the hazard. SP effort is a flow variable, such that only current efforts influence the probability of introduction, as might be observed with a visual inspection program. We also assume $\psi_{N}(N, z)<$ 0: the system can protect itself naturally, providing NICP. We make no assumptions about the signs of $\psi_{N N}$ and $\psi_{N z}$, as natural processes often involve complex, nonlinear interactions that 
generate convex-concave relations (Dasgupta and Maler 2003). The probability the system has evaded invasion to time $t$ is given by the survivor function $s(t)=e^{-\int_{0}^{t} \psi(N(v), z(v)) d v}=e^{-y}$, where $y(0)=0$, so $s(0)=1$.

The cost of reducing $h$ and investing in NICP is the opportunity cost of foregone $B(N)$. The unit cost of SP is unity, so that $z$ also represents total expenditures on SP. Given this specification, net benefits in each period $t<T$ are $B(N) h-z$.

Let $E$ be the expectations operator reflecting uncertainty about $T$. Ex ante efficient management is then defined as the solution to:

$$
\max _{h, z} E\left\{\int_{0}^{T}[B(N) h-z] e^{-r t} d t+e^{-r T} W(N(T))\right\}
$$

subject to $N(0), 0 \leq h \leq h^{\max }$ (where $h^{\max }$ is the maximum control level), $z \geq 0$, and the equation of motion in Equation (1). Problem (2) can be re-written as (see Appendix A2 for details, Reed and Heras 1992; Horan and Fenichel 2007; Finnoff et al. 2013):

$$
S N B=\max _{h, z}\left\{\int_{0}^{\infty}[B(N) h-z+\psi(N, z) W(N)] e^{-r t-y} d t\right\}
$$

subject to $N(0)$, the control constraints, and the equations of motion (1) and

(4) $\dot{y}=\psi(N, z), y(0)=0$.

The conditional, current-value Hamiltonian (henceforth, simply referred to as the Hamiltonian) associated with (3) is

$$
\begin{gathered}
H=B(N) h-z+\psi(N, z) W(N)+\lambda[g(N)-h]-\psi(N, z) \rho \\
=B(N) h-z-D(N, z, \rho)+\lambda[g(N)-h]
\end{gathered}
$$

where $\lambda>0$ is the co-state for $N, \rho>0$ is the co-state associated with $-y$, and $D(N, z)=$ $\psi(N, z)[\rho-W(N)]$. The term $D(N, z, \rho)$ represents the expected net cost of an invasion. This is because, as we show below in equation (9), $\rho$ is the present value of ex ante expected net benefits, so that 


$$
\gamma=\rho-W
$$

is the net economic cost of transitioning to the ex post outcome. At time $T$, society ceases to receive ex ante expected net benefits of $\rho$ and instead earns the ex post value of $W$. If $\gamma>0$, then there is value associated with trying to help the natural system prevent an invasion. If $\gamma<0$, then there is value to letting the invasion occur.

The concavity of $H$

Before optimizing, we examine whether problem (3) is likely to exhibit a global optimum. The easiest way to ensure such an outcome is to verify that the Mangasarian sufficiency conditions are satisfied, which for our model requires $H(N, h, z, \lambda, \rho)$ to be concave in $N$ and the controls $h$ and $z$ when $\lambda(t)=\lambda^{*}(t)$ and $\rho(t)=\rho^{*}(t)$ (Caputo 2005). As $H$ is linear in $h$, we focus on $N$ and $z$ and examine whether $H$ is concave in these variables. If not, there may be multiple, locally ex ante optimal solutions such that ex ante multi-stability exists in addition to ex post multistability. The following proposition addresses the concavity of $H$ :

Proposition 2. If $D(N, z, \rho)$ is convex in $N$ and $z$ then $H(N, h, z, \lambda, \rho)$ is concave in $N$ and $z$. Otherwise, if $D(N, z, \rho)$ is not convex, either due to (i) $W(N)$ being non-concave, (ii) the hazard rate $\psi(N, z)$ being non-convex due to the effects of $N$ (so that there are not diminishing returns to reducing the hazard of invasion), (iii) $N$ and $z$ being substitutes in reducing the hazard, or (iv) expected losses $\gamma$ being negative, then $H(N, h, z, \lambda, \rho)$ may not be concave in $N$ and $z$ and there is at least the potential for local optima.

Proof: See Appendix A3. 
Proposition 2 indicates that the Hamiltonian would be concave if there were no chance of invasion, and so only factors associated with the invasion create the potential for nonconvexities. Specifically, the potential for ex ante non-convexities depends on the role of $N$ in providing NICP. A non-concave $W(N)$ (condition i) may arise due to the native population's ability to protect itself from an invader that has been introduced. For instance, if a larger $N$ provided no (absolute) protection ex post, then a basin for the good (bad) equilibrium will not emerge; ex post multi-stability does not arise and $W(N)$ would be concave. A non-convex hazard (condition ii) arises if nonlinear natural processes cause a larger native population to generate increasing returns to reducing the hazard, at least for some $N$. Natural processes may also cause the native population to substitute for anthropogenic SP in reducing the hazard (condition iii). Finally, the ability of the native population to prosper from an invasion (condition iv), at least for some $N$, will be driven by natural processes affecting NICP.

None of the conditions (i) - (iv) are satisfied in prior resource "collapse" models, which make assumptions to guarantee $D$ is convex (e.g., Ranjan et al. 2008; Reed and Heras 1992). This suggests that NICP arises fairly trivially, if at all, in prior work.

\section{Optimality conditions}

We can write the Lagrangian associated with (5) and the control constraints as

$$
\begin{aligned}
L=B(N) h-z- & \psi(N, z)[\rho-W(N)]+\lambda[g(N)-h] \\
& +\mu^{h \min } h+\mu^{h \max }\left[h^{\max }-h\right]+\mu^{z \min _{Z}}
\end{aligned}
$$

where $\mu^{h \min } \geq 0, \mu^{h \max } \geq 0$, and $\mu^{z \min } \geq 0$ are the Lagrangian multipliers associated with the constraints $h \geq 0, h \leq h^{\max }$, and $z \geq 0$, respectively.

We now examine the necessary conditions for optimality, keeping in mind that non- 
convexities may be present. We begin with the adjoint conditions for the managed state $N$ and integrated hazard $y$, which are (Reed and Heras 1992):

$$
\begin{aligned}
& \dot{\lambda}=\left[r+\Psi(N, z)-g_{N}\right] \lambda-B_{N}(N) h-\psi_{N}(N, z)[W(N)+\rho]-\Psi(N, z) W_{N}(N) \\
& \dot{\rho}=[r+\Psi(N, z)] \rho-[B(N) h-z+\psi(N, z) W(N)]
\end{aligned}
$$

We examine condition (7) in detail below. Adjoint condition (8) has the solution

$$
\rho(t)=\int_{t}^{\infty}[B(N) h-z+\psi(N, z) W(N)] e^{-r(s-t)-y} d s
$$

Relation (9) indicates $-\rho(t)>0$ is the expected present value of net benefits from the current time onwards, or the ex ante value of an optimally managed system facing the threat of invasion.

The optimality condition for control of the managed stock is

$$
\frac{\partial L}{\partial h}=B(N)-\lambda+\mu^{h \min }-\mu^{h \max }=0 ; h \mu^{h \min }=0 ;\left[h^{\max }-h\right] \mu^{h \max }=0
$$

Condition (10) indicates that control occurs at a maximum rate when the current marginal net benefits of control exceed the marginal user cost, $B(N)>\lambda$; control ceases when the marginal user cost exceeds the current marginal net benefits of control, $B(N)<\lambda$; and the singular solution, denoted $h^{*}$, is pursued when these marginal net benefits and costs are equal, $B(N)=\lambda$.

Finally, the optimality condition for prevention effort, or anthropogenic insurance, is

$$
\frac{\partial L}{\partial z}=-1-\psi_{z}(N, z) \gamma+\mu^{z \min }=0 ; z \mu^{z \min }=0
$$

Our assumptions about $\psi_{z}$ imply that an interior solution arises when $\gamma>0$ : investments in selfprotection are made to augment natural risk reduction, with investment optimally occuring up to the level that balances the marginal cost of self-protection with the expected marginal reduction in losses, whenever establishment by the invader yields losses. Otherwise, if $\gamma \leq 0$ then $z=0$ is optimal. That is,

$$
\gamma=\frac{-1}{\psi_{z}} \geq 0 \text { iff } z \geq 0 \text { and } \mu^{z \min }=0 ; \gamma<0 \text { iff } z=0 \text { and } \mu^{z \min }>0
$$


Condition (12) indicates that some amount of investment in self-protection will occur to prevent costly invasions, regardless of whether some investment is also being made in natural insurance.

Conditions (11) and (12) indicate there are a variety of cases to consider. We simplify matters by primarily focusing on the singular solution for harvests, which leaves two cases to consider within this context: when it is optimal to self-protect, $z>0$, and when it is optimal to let the managed system protect itself, $z=0$. We examine each case in turn.

Case 1: $z \geq 0, \gamma \geq 0, \mu^{z \min }=0$, with $h=h^{*}$

With prevention, $z \geq 0$, condition (12) implies the following relation for $\gamma$

$$
\gamma(N, z)=\frac{-1}{\psi_{z}(N, z)} \geq 0
$$

Equation (A16) in Appendix A4 shows that the relation $\gamma(N, z)=\frac{B(N) g(N)-z-r W(N)}{r+\psi(N, z)}$ must also hold in the singular outcome. Rearranging this relation yields

$$
[B(N) g(N)-z-\psi(N, z) \gamma(N, z)]-r W(N)=r \gamma(N, z)
$$

The bracketed term on the left-hand-side (LHS) of relation (14) is the Hamiltonian in the singular outcome ( since $h(N)=g(N)$ is shown below to be the singular value $h^{*}$ ), which we show in Appendix A4 equals $r \rho$, or the flow value (dividend) of the expected net present value accruing when the invasion is prevented. The term $r W(N)$ is the expected flow value accruing after the invasion. If society could choose the invasion time $T$, the difference between these values — and hence $\gamma$-would optimally be zero when an invasion occurs. Society would then be indifferent to transitioning to the invaded regime, so that equation (14) with $\gamma=0$ is a transversality condition for the invasion date $T .{ }^{10}$ Alternatively, it is optimal to continue in the

\footnotetext{
${ }^{10}$ The solution $\gamma=0$ is also optimal in a non-random optimal stopping problem with $\psi=0$ (i.e., when the end date of the initial management phase, $T$, is deterministic and chosen optimally).
} 
non-invaded regime when the LHS is positive, so that $\gamma>0$; it is optimal to allow the invasion to occur when $\gamma<0$.

Although condition (14) is akin to a transversality condition, this condition is not associated with actively choosing the time $T$ at which the invasion will occur. This is because the time of invasion is uncertain, requiring a modification of the notion of a transversality condition. Consider the following alternative form of condition (14), which we interpret as a risk-adjusted transversality condition

$$
B(N) g(N)-z-[r+\psi(N, z)] \gamma(N, z)=r W(N)
$$

The LHS of relation (15) represents the current net benefits accruing in the singular outcome when the invasion is prevented, less the costs of prevention and risk-adjusted capital losses from invasion. The RHS is the expected flow value accruing after the invasion. Condition (15) optimally holds at each potential date for invasion, in contrast to a standard transversality condition that only holds in the terminal period. Given the current value of $N$, the mechanism for ensuring (15) is satisfied is the same as the mechanism for influencing the date of invasion, $T$ : it is the choice of $z$. Accordingly, condition (15) implicitly defines the optimal level of selfprotection, $z$.

To understand the optimal choice of $z$, first note that both $z$ and $\gamma$ are positive in condition (15), implying that $B(N) g(N)>r W(N)$ in case 1: the net benefits earned when there is no risk from establishment outweigh the expected dividends after establishment. Some of the difference between $B(N) g(N)$ and $r W(N)$ is reflected by the risk-adjusted capitalized losses that society is subject to ex ante, $[r+\psi(N, z)] \gamma(N, z)$. The residual difference is optimally invested in selfprotection:

$$
z=B(N) g(N)-[r+\psi(N, z)] \gamma(N, z)-r W(N)
$$


Denote the implicit solution to (16) by the feedback relation $z(N)$, which can be substituted into (13) to yield $\gamma(N) \equiv \gamma(N, z(N))$. The optimal investment $z(N)$ ensures society prefers continuing to help the managed system prevent the invasion. Indifference between whether society invests in self-protection (or not) occurs when $B(N) g(N)=r W(N)$, so that $z(N)=0$ (with $\mu^{z \text { min }}=0$ ) and $\gamma(N)=-1 / \psi_{z}(N, 0) \rightarrow 0$. The indifference relation $z(N)=0$ defines a boundary for case 1 , as values of $N$ such that $z(N)<0$ imply a corner solution for $z$ with $\mu^{z \min }>0$, and hence $\gamma(N)<0$ (which we examine in the following section).

We use a numerical example to illustrate some of the results as we move forward. Functional forms and parameter values, defined in Appendix A5, have been selected such that only conditions (i) and (iv) from Proposition 2 may lead to ex ante non-convexities.

Specifically, $W$ is convex and its large values when $N$ is large mean that $\gamma$ could be negative for large values of $N$ (note that $\psi$ is convex with $\psi_{N Z}=0$, so that conditions ii and iii in Proposition 2 cannot arise). The relation for $W$ is representative of the results in Finnoff et al. (2010) and in Horan et al. (2011). ${ }^{11}$

The first result we examine numerically is the indifference boundary $z(N)=0$ such that $\gamma(N)=0$ and $B(N) g(N)=r W(N)$. Figure 1 depicts the relations $B(N) g(N)$ and $r W(N)$ to determine values of $N$ such that $z(N)>0, z(N)=0$, and $z(N)<0$. We have already indicated that $W(N)$ is increasing and convex. The relation $B(N) g(N)$, which represents net revenues in the singular solution, is non-negative and concave along the domain $\left[N^{\pi=0}, K\right]$, where $N^{\pi=0}$ is the value of $N$ for which the net value of harvests is zero (concavity arises arises due to the combination of logistic growth and Schaefer harvesting costs that we use in our numeric example). Figure 1

\footnotetext{
${ }^{11}$ We have specified $W(N)$ rather than all of its sub-components, for simplicity. Our decisions about the shape of $W(N)$, both for our baseline scenario as well as for a variant analyzed below, are in accordance with our earlier discussion of the properties of $W(N)$ as arising from the various underlying components.
} 
indicates that a range of $N$ exists such that $z(N), \gamma(N)>0$ and hence $B(N) g(N)>r W(N)$, bounded by lower and upper threshold values of $N$ such that $z(N)=0$. Specifically, $z(N)>0$ for $N \in$ $\left(\widetilde{N}^{L}, \widetilde{N}^{H}\right)$ where $N^{\pi=0}<\widetilde{N}^{L}<\widetilde{N}^{H}<K$ such that $B\left(\tilde{N}^{j}\right) g\left(\tilde{N}^{j}\right)=r W\left(\tilde{N}^{j}\right)$ for $j \in\{L, H\} .{ }^{12}$ The result is that case $1\left(z \geq 0, \gamma \geq 0\right.$ with $\left.\mu^{z \min }=0\right)$ can only arise for $N \in\left(\widetilde{N}^{L}, \widetilde{N}^{H}\right)$. Finally, we derive the singular value of $N$ for case 1 . In a singular outcome, condition (12) requires $\lambda=B(N)$. We can time differentiate this relation and then use (9) to obtain

$$
r=\underbrace{\left[g_{N}(N)+\frac{B_{N}(N) g(N)}{B(N)}\right]}_{R^{R F}(N)}+\underbrace{\left[\frac{-\psi_{N}(N, z(N)) \gamma(N, z)}{B(N)}-\frac{\psi(N, z(N))\left[B(N)-W_{N}(N)\right]}{B(N)}\right]}_{R^{N I C P}(N)}
$$

Condition (17) is a modified golden rule for resource management, which directs how the managed stock $N$ ought to be managed as a natural asset. The condition seeks a balance between the required rate of return, $r$ (what a market asset would return), to the own rate of return to conservation (the rate of return from holding the natural asset, given by the RHS with all economic values normalized by the marginal value of the managed stock). The first RHS quantity in brackets comprises two terms: the marginal growth of the resource plus the marginal cost savings from conservation. These terms are standard in resource models (Clark 2005), and we denote this quantity as the risk-free own rate of return, or $R^{R F}(N)$. In standard resource models, the golden rule condition $r=R^{R F}(N)$ determines the risk-free singular solution for $N$, which we denote as $N^{R F}$.

The second RHS quantity in brackets, denoted $R^{N I C P}(N)$, represents the own rate of return due to the effects of conservation in managing invasion risk: the rate of return to NICP. Indeed, noting that $d \gamma / d N=d \rho / d N-W^{\prime}(N)$ with $d \rho / d N=\lambda=B(N)$ (since $\rho$ is the expected net benefits in

\footnotetext{
${ }^{12}$ There will only be two threshold values given our assumptions that lead to $W(N)$ monotonically increasing. More than two thresholds could arise if $W(N)$ was non-monotonic.
} 
the pre-invasion interval), $R^{N I C P}(N)$ can be re-written as $-\left[D_{N}(N, \rho(N), z(N))+D_{\rho}(N, \rho(N), z(N))\right.$ $\left.\rho^{\prime}(N)\right] / B(N)$ : the normalized marginal effect of $N$ on reducing the expected economic losses from invasion, holding $z$ constant. Recall that NICP refers to the ability of the natural ecosystem to protect itself, by both lowering the chance the ecosystem transitions to being invaded and lowering the expected losses that occur if the ecosystem becomes invaded. The first term in $R^{N I C P}(N),-\psi_{N} \gamma / B>0$, reflects the first component of NICP: the value of lowering the chance the ecosystem transitions. Specifically, this term is the (normalized) marginal effect of $N$ on reducing expected losses, holding $\gamma$ fixed. Since $\psi_{N}<0$, a more abundant managed stock reduces invasion risks, increasing $R^{N I C P}(N)$.

The second term in $R^{N I C P}(N)$ reflects the second component of NICP: lowering the expected losses that occur if the ecosystem becomes invaded. These losses arise here as potential risks of reduced natural capital values at the date of invasion. If the invasion date $T$ was deterministic, then $N(T)$ would be chosen to satisfy the standard transversality condition for $N, \lambda(T)=B(N(T))=W_{N}(N(T))$. This condition is unlikely to be satisfied, however, when the invasion date $T$ random; the values $B(N(T))$ and $W_{N}(N(T))$ will likely deviate at each possible date for $T$. The expected difference, $\psi(N)\left[W_{N}(N)-B(N)\right]$, is the expected marginal capital gain or loss from invasion. An efficiency gain occurs if $B(N)<W_{N}(N)$ when $T$ occurs, increasing the return to $N$. The opposite occurs if $B(N)>W_{N}(N)$ at $T$. This means conservation does not necessarily increase this component of NICP: the net effect of conservation on expected losses depends on ex post impacts relative to ex ante impacts. The net effect on NICP is more likely to be positive when larger $N$ has a greater impact on increasing the chance the ex post system alights in the good basin of attraction, and if the expected gain from such an outcome is sufficiently large. 
Condition (17) is only a function of the managed stock, $N$, providing a rule for the intertemporal management of $N$. Rearranging this condition can facilitate comparison with riskfree models. Specifically, we can write condition (17) as $r-R^{R F}(N)=R^{N I C P}(N)$, where the RHS is the return to NICP, and the LHS is the required return to managing invasion risk (i.e., the required return $r$ net of the risk-free return that has been generated). If the return to NICP is positive, then $r>R^{R F}(N)$ and the singular value of $N$ is likely smaller than $N^{R F}$. Multiple equilibria may be possible within the domain $\left(\widetilde{N}^{L}, \widetilde{N}^{H}\right)$. Denote equilibrium $i$ as $N^{i}$. As $N^{i}$ is unchanging over time, $h^{i}=g\left(N^{i}\right)$ and the previously-defined values of $z\left(N^{i}\right), \rho\left(N^{i}\right)$, and $\gamma\left(N^{i}\right)$ are also constant in the singular outcome.

Figures 2-3 plot the curves $r-R^{R F}(N)$ and $R^{N I C P}(N)$ for our numerical example (Appendix A5), along with other relations as indicated. Absent invasion risks, a unique risk-free singular stock level occurs at $N^{R F}$, where $r-R^{R F}(N)=0$ (Figure 2). $N^{R F}$ is relatively small due to $B(N)$ being relatively large.

Now consider the impact of invasion risks for values of $N \in\left(\widetilde{N}^{L}, \widetilde{N}^{H}\right)$ such that $\gamma>0$. Within this interval, relation $\psi(N, 0)$ in Figure 2 represents the hazard in the absence of selfprotection. This hazard is a declining function of $N$ due to the managed stock providing NICP. However, a larger $N$ also initially yields $d \gamma / d N=B(N)-W^{\prime}(N)<0$, reducing NICP: the loss due to an invasion is initially increasing in $N$ when $N$ is small, as a small $N$ is more likely to place the invaded system in the bad basin of attraction. The net effect is that $R^{N I C P}(N)$ initially declines in $N$ and quickly becomes negative. As the risk of invasion is greatest when $N$ is small, there is significant value in investing in $z$ (SP) to offset this risk. Relative to $\psi(N, 0)$, the curve $\psi(N, z(N))$ shows how SP augments the risk reduction offered by NICP. The investment level $z(N)$ is shown in Figure 3. 
Once $N$ becomes sufficiently large, $R^{N I C P}(N)$ increases again and becomes positive as $d \gamma / d N>0$ : a larger $N$ reduces losses by increasing the likelihood of being in the good basin. The greater this likelihood, the fewer benefits from preventing an invasion, and $\mathrm{z}(N)$ is reduced (Figure 3).

The singular solutions, denoted $N^{A}$ and $N^{B}$, occur at the intersections of the curves $r-$ $R^{R F}(N)$ and $R^{N I C P}(N)$ within the positive orthant. The curve $R^{R}(N)$ is non-monotonic, yet increasing and convex over much of the relevant range. Given the increasing and concave $r-$ $R^{R F}(N)$ curve (which is common in standard resource models), it is the increasing and convex nature of $R^{N I C P}(N)$ that enables two equilibria to arise. Given that our modeling assumptions rule out conditions (ii) and (iii) from Proposition 2, and that the outcome $\gamma>0$ for case 1 rules out condition (iv), it is clear that ex post non-convexities drive the multiple ex ante equilibria.

The first equilibrium, $N^{A}$, involves a smaller risk-free own rate of return, $R^{R F}(N)$, relative to the risk-free equilibrium (as indicated by the intersection of $r-R^{R F}(N)$ and $R^{N I C P}(N)$ above the horizontal axis at $N^{A}$ ) to offset the positive return to NICP. This equilibrium is locally stable because $R^{N I C P}(N)>r-R^{R F}(N)$ to the left of $N^{A}$, so that the total own rate of return to conservation $\left(R^{R F}(N)+R^{N I C P}(N)\right)$ exceeds the discount rate, whereas the opposite holds to the right of $N^{A}$. As might be expected, $N^{A}>N^{R F}$ since a larger $N$ provides NICP and reduces invasion risks in this example. However, the difference $N^{A}-N^{R F}$ is slight because SP is used more extensively at $N^{A}$ than NICP (see $z(N)$ plotted in Figure 3), as described above.

The second equilibrium, $N^{B}$, is unstable since $r-R^{R F}(N)$ cuts $R^{N I C P}(N)$ from below. At this equilibrium, $N$ is at a high enough level that its risk reduction almost perfectly substitutes for $z$ (Figure 3). There is very little investment in SP at this point because $\gamma$ is small even without much investment in SP. While unstable, equilibrium $N^{B}$ is not necessarily a Skiba point, but the 
instability associated with this point does suggest that additional, possibly locally stable, equilibria may exist for $N>\widetilde{N}^{H}$, with $z=0$ and $\gamma<0$. We now explore this case.

Case 2: $z=0, \gamma<0$ with $h=h^{*}$

Suppose $z=0$ is optimal as a corner solution with $\mu^{z \text { min }}>0$, in which case condition (12) implies $\gamma<0$. In this case, the feedback relation $\gamma(N)$ stems from the risk-adjusted transversality condition (15) with $z=0$ : the value of $\gamma$ must adjust as follows to satisfy (15),

$$
\gamma(N)=\frac{B(N) g(N)-r W(N)}{r+\psi(N, 0)}<0,
$$

since $z$ is now unavailable to ensure (15) holds. The denominator is positive, and so condition (18) requires $r W(N)>B(N) g(N)$ : the expected dividends if an invasion occurs outweigh the flow of control benefits. Society prefers to not prevent the invasion $(z=0)$ because the managed state is in such a pristine state that it provides enough natural insurance to eliminate the consequences of invasion, without any need for human intervention. An outcome with $\gamma<0$ in this case indicates the value of the pristine managed state a larger $N$ substitutes for prevention and provides a highly valued ex post $W(N)$.

The modified golden rule relation in this situation becomes

$$
r=\left[g_{N}(N)+\frac{B_{N}(N) g(N)}{B(N)}\right]-\frac{\psi_{N}(N, 0) \gamma(N, 0)}{B(N)}-\frac{\psi(N, 0)\left[B(N)-W_{N}(N)\right]}{B(N)}
$$

With $\gamma<0$, the third RHS term in (19) has the opposite sign as in expression (17). Specifically, the third RHS term in (19) is negative, reducing the return to NICP: other things equal, a smaller $N$ increases the likelihood of invasion, which is desirable since $\gamma<0$. This negative term creates diminishing returns to NICP, causing the curve $R^{N I C P}(N)$ to become concave and intersect with the curve $1-R^{R F}(N)$ to create a second stable solution, denoted $N^{C}>\widetilde{N}^{H}$, along with a constant singular harvest $h^{C}=g\left(N^{C}\right)$. 


\section{The Complete Numerical Solution}

Equilibria $N^{A}$ and $N^{C}$ are both optimality candidates, with each having the potential to be locally or globally optimal. The optimality of the equilibria depends on each equilibrium's basin of attraction, which in turn depends on the transition paths from the initial stock value $N(0)$. Condition (10) indicates the transition to a particular equilibrium involves choosing $h$ to move as quickly as possible to the desired singular solution along a most rapid approach path (MRAP). The speed of transition, and hence the benefits along a particular transition path, depend on how fast the adjustment can occur, such as how fast the stock regenerates or the magnitude of $h^{\max }$. Slower transitions are likely to result in the candidate equilibria being locally optimal, with a Skiba point separating the basins of attraction (Brock and Starrett 2003). We have drawn Figures 2-3 with the Skiba point occurring at $N^{B}$, although this need not be the case as the Skiba point could be greater or lesser than this value, depending on the speed of transition.

As described above, equilibrium $N^{A}$ reflects a somewhat depleted managed stock in response to large benefits associated with harvesting. The depleted stock creates invasion risks, with the ex post invaded outcome yielding a potentially poor economic (and ecological) outcome. In response to this risk it is optimal to slightly increase $N$ relative to the risk-free case (i.e., $N^{A}$ is slightly larger than $N^{R F}$ ), in combination with investments in $z$, to reduce invasion risks. But while the invasion likelihood $\psi$ has been reduced, the comparatively small value of $N^{A}$, combined with $W_{N}>0$, yields relatively large risks of a poor ex post outcome. This is a risk worth taking when $N(0)$ is sufficiently small (less than the Skiba value), $\gamma(N)$ is relatively large, and $\psi(N, z(N))$ is relatively small in the neighborhood of $N^{A}$.

Equilibrium $N^{C}$ yields fewer singular harvest benefits, $B(N) g(N)$, than $N^{A}$ (Figure 1) for 
lower singular harvests (Figure 3), but the relatively large managed stock in this case increases the likelihood that an invasion will result in the system alighting in a good ex post outcome. Of course, it is not guaranteed that the ex post system will end up in the basin associated with the good equilibrium; society may be unlucky and end up in the other basin. However, this is also a risk worth taking when $N(0)$ is sufficiently large and when $W(N)$ and $\psi(N, 0)$ are sufficiently large in the neighborhood of $N^{C}$. The requirement of a large $W(N)$ is straightforward to demonstrate. A sufficiently large $\psi(N, 0)$ is required due to the relatively large opportunity costs of remaining at a large $N$ in the ex ante system: society expects to be better off, at least for large values of $N$, by transitioning to the ex post outcome. Equilibrium $N^{C}$ disappears and $N^{A}$ becomes globally optimal if $\psi$ is reduced so that invasion becomes sufficiently less likely $\forall N$, or if $W(N)$ is smaller for larger values of $N$ - particularly if an invasion is costly for large $N$.

\section{Conclusion}

Risks posed by invasive species can be reduced by natural or human risk reduction actions, or both. Our model demonstrates how ex ante management through a mix of natural and human risk reduction provides opportunities to influence the ex post conditions and ex post optimal outcomes. We have shown this by extending the Poisson "collapse" model of Reed and Heras (1992) to allow for direct management of self-protection and indirect management of naturalinsurance-cum-protection, to endogenize initial conditions and influence ex post multi-stability.

The endogenous risk of an invasion modifies the standard transversality conditions from deterministic models. Transitions to invaded states are probabilistically managed by the terminal stock condition being embedded in the golden rule equation for resource management, adding an additional layer of complexity to the penalties and rewards for conservation. 
We find that an ex ante system that is convex and uniquely stable without invasion risk may become non-convex and multi-stable in the presence of endogenous invasion risks and ex post multi-stability. This result arises because ex post non-convexities, and the uncertainties associated with the invasion and its magnitude, can create ex ante non-convexities that generate multiple optimality candidates.

In our numeric example, for a wide range of initial managed states, including those in the vicinity of optimal management in the absence of invasion risk, a mix of natural and human risk reduction is required. However, relatively pristine initial managed states, near the unmanaged equilibrium, can protect themselves. The key implication is that the optimal investments prior to invasion are tied to the likely outcome after invasion. Investing in natural risk reduction now, while potentially costly, can lead to a "desirable" ex post steady state. Investing in selfprotection now can generate sizable benefits today, but this puts society at risk of having to rely on costly self-protection into perpetuity. 


\section{Appendix. Some Mathematical Results}

\section{Result A1. Mathematical underpinnings of $W(N)$ and proof of Proposition 1}

Immediately after an invasion, managers may pursue one of two candidate strategies, indexed by $i \in\{g, b\}$. Candidate strategy $g$ involves pursuing the most economically beneficial path to the good equilibrium, whereas candidate strategy $b$ involves pursuing the most beneficial path to the bad equilibrium. Define the present value of net benefits associated with strategy $i$ by $v^{i}(N(T), I)$, where $I \in[0,1]$ is variable positively correlated to the adverse ecological pressure initially created by an invasion ( $I$ is not time-indexed since the invasion only occurs once, and there are no exogenous dynamics for $I$ ). We make the usual assumptions with respect to $N, v_{N}^{i}>$ $0, v_{N N}^{i}<0$. We also assume that $v_{I}^{i}<0$, at least when $N$ is sufficiently small due to the invader adversely impacting the managed stock in this case. We allow for the possibility that $v_{I}^{i}>0$ when $N$ is sufficiently large, as the invader might serve as prey for the managed stock in that case.

Managers will optimally pursue the good strategy when $v^{g}(N(T), I)>v^{b}(N(T), I)$, such that the post-invasion system is initially in the good basin of attraction. The bad basin of attraction is optimally pursued when $v^{g}(N(T), I)<v^{b}(N(T), I)$, such that the post-invasion system is initially in the bad basin of attraction. The post-invasion system is initially on the Skiba threshold when $v^{g}(N(T), I)=v^{b}(N(T), I)$. For a given $N(T)$, there is a critical value $I^{*}(N(T))$ such that $v^{g}\left(N(T), I^{*}(N(T))\right)=v^{b}\left(N(T), I^{*}(N(T))\right)$, with $v^{g}(N(T), I)>v^{b}(N(T), I)$ for $I<I^{*}(N(T))$. Multi-stability occurs when $I^{*}(N(T)) \in(0,1)$ for at least some $N$. We simplify the math by assuming $I^{*}(N(T)) \in(0,1) \forall N$; multiple basins of attraction are possible for each $N$, with the realized basin for the ex post system depending on $I$. Suppose $I_{N}^{*}=\left(v_{N}^{g}-v_{N}^{b}\right) /\left(v_{I}^{b}-\right.$ $\left.v_{I}^{g}\right)>0$, so that the basin of attraction for the good equilibrium expands as $N$ is increased. This 
is indicative of higher levels of the managed state providing natural protection against invasion losses. Both the numerator and denominator of $I_{N}^{*}$ are assumed positive. The relation $v_{N}^{g}-v_{N}^{b}>$ 0 means a larger $N$ has a bigger marginal impact in the good state. The relation $v_{I}^{b}-v_{I}^{g}>0$ means that greater environmental pressure has a larger (i.e., more negative) marginal impact on the good state.

Ex ante, uncertainty exists about magnitude of the invasion and its impact, such that $z$ is random ex ante. Define $\phi(I, N(T))$ as the pdf of $I$, conditional on an invasion having occurred. This pdf is conditional on $N(T)$ because the state of the native system may affect the initial level of environmental pressure after an invasion. We assume increases in $N$ make larger values of $I$ less likely, in the sense of first-order stochastic dominance. In terms of the pdf, a larger $N$ shifts the probability mass to the left. This is the case when the invader competes with natives for resources or serves as a prey for the natives, such that a more pristine setting prevents the native system from being dominated by the invader (e.g., a relatively pristine rangeland system with perennial grasses prevents the invaded system from switching to primarily invasive annual grasses).

From an ex ante perspective, and dropping the time indices for simplicity, the expected net benefits of the ex post outcome are

$$
W(N)=\int_{0}^{I^{*}(N)} v^{g}(N, I) \phi(I, N) d I+\int_{I^{*}(N)}^{1} v^{b}(N, I) \phi(I, N) d I
$$

Ex post expected net benefits depend on the managed state, with $W(0) \leq 0$. It is easily verified that $W(N)$ is an increasing function:

$$
\begin{aligned}
W_{N}(N)= & \int_{0}^{I^{*}(N)} v_{N}^{g}(N, I) \phi(I, N) d I+\int_{I^{*}(N)}^{1} v_{N}^{b}(N, I) \phi(I, N) d I \\
& +\int_{0}^{I^{*}(N)} v^{g}(N, I) \phi_{N}(I, N) d I+\int_{I^{*}(N)}^{1} v^{b}(N, I) \phi_{N}(I, N) d I>0
\end{aligned}
$$


The first two right-hand-side (RHS) terms in (A2) are positive due to the assumption that $v_{N}^{i}>$ 0 , and the second line of (A2) is positive due to our assumption of first order stochastic dominance: a larger $N$ makes the good state more likely, producing greater values on average.

We can now prove Proposition 1:

Proposition 1. $W(N)$ is concave in the absence of ex post multi-stability or ex ante uncertainty about $I$. However, either ex post multi-stability or ex ante uncertainty about $I$ (or both) may cause $W(N)$ to be convex over a range of $N$.

Proof. Consider the second derivative of $W(N)$ :

$$
\begin{aligned}
& W_{N N}(N)= \int_{0}^{I^{*}(N)} v_{N N}^{g}(N, I) \phi(I, N) d I+\int_{I^{*}(N)}^{1} v_{N N}^{b}(N, I) \phi(I, N) d I \\
&+2\left[\int_{0}^{I^{*}(N)} v_{N}^{g}(N, I) \phi_{N}(I, N) d I+\int_{I^{*}(N)}^{1} v_{N}^{b}(N, I) \phi_{N}(I, N) d I\right] \\
&+\left[v_{N}^{g}\left(N, I^{*}(N)\right)-v_{N}^{b}\left(N, I^{*}(N)\right)\right] \phi_{N}\left(I^{*}(N), N\right) I_{N}^{*}(N)
\end{aligned}
$$

The first line on the RHS of (A3) is negative due to the concavity of $v^{i}$. The second line of (A3) is positive due to a larger $N$ shifting the probability mass towards the good basin of attraction and since $v_{N}^{g}(N, I)-v_{N}^{b}(N, I)>0$. The sign of the third line of (A3) is ambiguous and will be the same as the sign of $\phi_{N}\left(I^{*}(N), N\right)$. The first line on the RHS of (A3) would be the only element of $W_{N N}(N)$, ensuring $W$ is concave, if not for ex post multi-stability or uncertainty about $I . W(N)$ will be convex (convex-concave) if the net value of the second and third lines of (A3) is positive and dominates the first line for all (some) $N$.

\section{Result A2. Derivation of SNB in Equation (3)}

Evaluate the expectations in Equation (2) of the main text by writing the interior terms using the indicator functions $i(t \leq T)$ which has the value 1 when $t \leq T$ and 0 otherwise, and the 
indicator function $j(t=T)$ which has the value 1 when $t=T$ and 0 otherwise

(A4) $\max _{h, z} E_{T}\left\{\int_{0}^{\infty} j(t \leq T)[B(N) h-z] e^{-r t} d t+\int_{0}^{\infty} j(t=T) e^{-r t} W\left(N_{T}\right) d t\right\}$

The expectation of $j(t \leq T)$ with respect to the distribution of $T$ is the survivor function $s(t)=$ $\exp \left(-\int_{0}^{t} \psi(N(v), z(v)) d v\right)=e^{-y}$, and the expectation of $i(t=T)$ is the density function $\psi(N, z) s(t)$. The hazard rate is the conditional probability of a regime shift, given one has not occurred already. The survivor rate is the probability of surviving up to point $T$. The survivor rate multiplied by the hazard rate is the probability of $T$ occurring at a given $t$. Substituting these definitions into (A4) gives

$$
\max _{h, z}\left\{\int_{0}^{\infty} e^{-y}[B(N) h-z] e^{-r t} d t+\int_{0}^{\infty} \psi(N(t), z(t)) e^{-y} e^{-r t} W\left(N_{T}\right) d t\right\}
$$

Combining terms this becomes

$$
\max _{h, z}\left\{\int_{0}^{\infty}[B(N) h-z+\psi(N(t), z(t)) W(N(T))] e^{-r t-y} d t\right\}
$$

\section{Result A3. Proof of Proposition 2}

Proposition 2. If $D(N, z, \rho)$ is convex in in $N$ and $z$ then $H(N, h, z, \lambda, \rho)$ is concave in $N$ and $z$.

Otherwise, if $D(N, z, \rho)$ is not convex, either due to (i) $W(N)$ being non-concave, (ii) the hazard rate $\psi(N, z)$ being non-convex due to the effects of $N$ (so that there are not diminishing returns to reducing the hazard of invasion), (iii) $N$ and $z$ being substitutes in reducing the hazard, or (iv) expected losses $\gamma$ being negative, then $H(N, h, z, \lambda, \rho)$ may not be concave in $N$ and $z$ and there is at least the potential for local optima.

Proof: Without the function $D(N, z, \rho)$, the Hamiltonian is given by $\left.H\right|_{D=0}=B(N) h-z+\lambda[g(N)-$ $h$ ]. This relation is linear in $z$, and it is concave in $N$ since $B$ and $g$ are concave in $N, \lambda>0$ along an optimal path (since $N$ is only a source of value in this problem), and the sum of two concave functions is concave (Simon and Blume 1994; Theorem 21.8). By the same logic, subtracting 
$D(N, z, \rho)$ from $\left.H\right|_{D=0}$, thereby producing $H$, is concave if $D$ is convex. But $D$ may not be convex. Convexity of $D$ in $N$ and $z$ requires

(A7a) $D_{z z}=\psi_{z z} \gamma>0$

(A7b) $D_{N N}=\psi_{N N} \gamma-2 \psi_{N} W_{N}-\psi W_{N N}>0$,

and $D_{N N} D_{Z Z}<D_{N Z}^{2}$, which can be written as

(A7c) $\left[\psi_{N N} \psi_{z z}-\psi_{N z}\right] \gamma^{2}+\left[-W_{N N} \psi_{z z}\right] \psi \gamma>2 \psi_{N} W_{N} \psi_{z z} \gamma-\left[\psi_{z} W_{N}\right]^{2}-2 \psi_{z} W_{N} \psi_{N z}$

Our assumptions about $\psi$ ensure condition (A7a) holds when $\gamma=\rho-W>0$, and is violated when $\gamma<0$. Condition (A7b) is satisfied when $W$ is concave and when either (a) $\psi_{N N}>0$ and $\gamma>0$ or (b) $\psi_{N N}<0$ and $\gamma<0$. Since $W$ may not be concave and the signs of $\psi_{N N}$ and $\gamma$ are unknown, condition (A7b) may not hold. Consider condition (A6c). The LHS of this condition is positive when $\psi$ is convex (which depends on the role of $N$, since $\psi_{z z}>0$ by assumption), $W$ is concave, and $\gamma>0$, and the RHS is negative when $\gamma>0$ and $\psi_{N z} \leq 0$, such that $N$ and $z$ are not substitutes in protecting the ecosystem from invasion. This special case guarantees condition $(\mathrm{A} 7 \mathrm{c})$ is satisfied. If $D$ is not convex, then concavity of the Hamiltonian in $N$ and $z$ is still possible and requires $H_{z z}=-D_{z Z}<0, H_{N N}=B_{N N}-D_{N N}+\lambda g_{N N}<0$, and $H_{N N} H_{z z}-H_{N Z}^{2}=H_{N N} H_{z Z}-$ $D_{N Z}^{2}>0$. These conditions are impossible to evaluate analytically without additional structure.

\section{Result A4. Derivation of $\rho$}

The time derivative of the present value of the optimized Lagrangian is:

$$
\begin{aligned}
-\frac{d\left[L e^{-r t-y}\right]}{d t}=-[ & \left.\frac{\partial L}{\partial h} \dot{h}+\frac{\partial L}{\partial z} \dot{z}+\frac{\partial L}{\partial N} \dot{N}+\frac{\partial L}{\partial y} \dot{y}+\frac{\partial L}{\partial \lambda} \dot{\lambda}+\frac{\partial L}{\partial \rho} \dot{\rho}+\sum_{i \in\{h \min , h \max , z \min \}} \frac{\partial L}{\partial \mu^{i}} \dot{\mu}^{i}\right] e^{-r t-y} \\
& -L e^{-r t-y}(-[r+\psi])
\end{aligned}
$$

Following Tahvonnen and Salo (1996), the necessary conditions can be used to write (A8) as 


$$
\begin{aligned}
-\frac{d\left[L e^{-r t-y}\right]}{d t}=-[0 & +0+([r+\psi] \lambda-\dot{\lambda}) \dot{N}+0+\dot{N} \dot{\lambda} \\
& +([r+\psi] \rho+B(N) h-z+\psi W(N)) \dot{y}+0+0+0] e^{-r t-y} \\
& +e^{-r t-y}([r+\psi][B(N) h-z+\psi W(N)+\lambda \dot{N}+\rho \psi+0+0+0]) \\
= & e^{-r t-y} r[B(N) h-z+\psi W(N)]
\end{aligned}
$$

Integrating both sides from any time $\tau$ onward yields

(A10) $-\int_{\tau}^{\infty} \frac{d\left[L e^{-r t-y}\right]}{d t} d t=r \int_{\tau}^{\infty} N B e^{-r t-y} d t=r S N B(\tau)$

where $N B=B(N) h-z+\psi W(N)$. Assuming $L(\infty)$ is finite, expression (A10) becomes

(A11) $L(\tau) e^{-r \tau-y(\tau)}-L(\infty) e^{-\infty-y(\infty)}=L(\tau) e^{-r \tau-y(\tau)}=r S N B(\tau)$.

Reed and Heras show that the following relation must also hold along an optimal path

(A12) $\rho(\tau)=e^{r \tau+y(\tau)} \frac{\partial S N B(\tau)}{\partial[-y(\tau)]}$

From (A11) and (A12), we can derive

$$
\rho(\tau)=e^{r \tau+y(\tau)}\left[\frac{L(\tau) e^{-r \tau-y(\tau)}}{r}\right]=e^{r \tau+y(\tau)}\left[\frac{H(\tau) e^{-r \tau-y(\tau)}}{r}\right]=\frac{N B(\tau)+\lambda(\tau) \dot{N}(\tau)+\rho(\tau) \dot{y}(\tau)}{r}
$$

The RHS of expression (A13) can be simplified using the first order conditions (and henceforth dropping the time notation)

$$
\begin{aligned}
\rho & =\frac{B(N) h-z+\psi(N, z) W(N)+\left[B(N)+\mu^{h \min }-\mu^{h \max }\right][g(N)-h]+\rho \psi(N, z)}{r} \\
& =\frac{-z+\psi(N, z) W(N)+\left[B(N)+\mu^{h \min }-\mu^{h \max }\right] g(N)-\mu^{h \min } \cdot 0+\mu^{h \max } h^{\max }+\rho \psi(N, z)}{r} \\
& =\frac{\left[B(N)+\mu^{h \min }\right] g(N)+\mu^{h \max }\left[h^{\max }-g(N)\right]-z+\psi(N, z) W(N)+\rho \psi(N, z)}{r}
\end{aligned}
$$

Solving (A14) for $\rho$ yields

$$
\rho=\frac{\left[B(N)+\mu^{h \min }\right] g(N)+\mu^{h \max }\left[h^{\max }-g(N)\right]-z+\psi(N, z) W(N)}{r+\psi(N, z)}
$$


Condition (A15) along with the relation $\gamma=\rho-W(N)$ can be used to derive

(A16) $\gamma=\frac{\left[B(N)+\mu^{h \min }\right] g(N)+\mu^{h \max }\left[h^{\max }-g(N)\right]-z-r W(N)}{r+\psi(N, z)}$

Note that $\mu^{h \min }=\mu^{h \max }=0$ in the singular outcome.

\section{A5. Numerical Model Parameterization}

The following specification has been used:

$g(N)=\alpha N(1-N / K)$

$B(N)=p-c / N$

$W(N)=w_{1} N^{2}-w_{2} N^{3}+w_{3} N^{4}$

$\psi(N, z)=0.24-0.01 N-0.05 z^{0.5}$

$\alpha=0.1, K=10, p=150, c=160, r=0.05, w_{1}=15, w_{2}=0.225, w_{3}=0.015$ 


\section{References}

Beavis, B. and M. Walker. 1983. Achieving Environmental Standards with Stochastic Discharges. Journal of Environmental Economics and Management 10: 103-111.

Bossenbroek, J. M., Nekola, J. C. \& Kraft, C. E. 2001. Prediction of long-distance dispersal using gravity models: zebra mussel invasion of inland lakes. Ecol. Appl. 11, 1778-1788.

Brock, W.A. and D. Starrett. 2003. Managing Systems with Non-Convex Positive Feedback. Environmental and Resource Economics 26: 575-602.

Brown, G., T. Patterson and N. Cain. 2011. The devil in the details: Non-convexities in ecosystem service provision. Resource and Energy Economics 33: 355-365.

Case, T.J. 1990. Invasion Resistance Arises in Strongly Interacting Species-Rich Model Competition Communities. Proceedings of the National Academy of Sciences of the United States of America 87: 9610-9614.

Chakraborty, S., C. Papageorgiou, and F.P. Sebastián. 2010. Diseases, Infection Dynamics, and Development. Journal of Monetary Economics 57: 859-872.

Chen, F., M. Jiang, S. Rabidoux, and S. Robinson. 2011. Public Avoidance and Epidemics: Insights from an Economic Model. Journal of Theoretical Biology 278: 107-119.

Clark, C.W. 2005. Mathematical Bioeconomics: The Optimal Management of Renewable Resources, John Wiley and Sons: New York.

Crocker, T.D. 1995. Ecosystem Functions, Economics, and the Ability to Function. Integrating Economic and Ecological Indicators, JW. Milon and J. Shogren, eds., Praeger, Westport, CT, $27-42$.

Crocker, T. D., and B. A. Forster. 1981. Decision Problems in the Control of Acid Precipitation: Nonconvexities and Irreversibilities. J. Air Pollution Control Assoc. 31:31-37. 
Crocker, T.D. and J.F. Shogren. 2001. Ecosystems as Lotteries, in Frontiers of Environmental Economics (H. Folmer, H.L. Gabel, S. Gerking and A. Rose, eds.), Cheltenham, UK and Edward Elgar, Northampton, MA, pp. 250271.

Dasgupta, P. and K.-G. Maler. 2003. The Economics of Non-Convex Ecosystems: Introduction. Environmental and Resource Economics 26: 499-525.

Davis, M.A., J.P. Grime, and K. Thompson. 2000. Fluctuating Resources in Plant Communities: A General Theory of Invasibility. Journal of Ecology 88: 528-534.

Drury, K.L.S. and D.M. Lodge. 2009. Using Mean First Passage Times to Quantify Equilibrium Resilience in Perturbed Intraguild Predation Systems. Theoretical Ecology 2.1: 41-51.

Ehrlich, I and G.S. Becker, 1972. Market Insurance, Self-Insurance, and Self-Protection. The Journal of Political Economy, 80(4): 623-648.

Fenichel, E.P. and R.D. Horan. 2007. Jointly-Determined Ecological Thresholds and Economic Tradeoffs in Wildlife Disease Management. Natural Resource Modeling 20: 511-547.

Fenichel, E.P., R.D. Horan, and J. Bence. 2010. Indirect Management of Invasive Species through Bio-Controls: A Bioeconomic Model of Salmon and Alewife in Lake Michigan. Resource and Energy Economics 32: 500-518.

Finnoff, D., J.F. Shogren, R.D. Horan, S.M. McDermott, and C. Sims. 2013. Economic Control of Invasive Species, pp.16-24 in Encyclopedia of Biodiversity, Second Edition, Volume 3 (S. Levin, ed). Waltham, MA: Academic Press.

Finnoff, D., A. Strong and J. Tschirhart, 2008. “A Bioeconomic Model of Cattle Stocking on Rangeland Threatened by Invasive Plants and Nitrogen Deposition.” American Journal of Agricultural Economics 90 (4): 1074-90. 
Finnoff D., M. A. Lewis and A. B. Potapov, 2010. Control and the Optimal Management of a Spreading Invader. Resource and Energy Economics, 32: 534-550.

Gilligan, C.A. and F. van den Bosch. 2008. Epidemiological Models for Invasion and Persistence of Pathogens. Annual Review of Phytopathology 46: 385-418.

Lee, K., 1998. Risk Aversion and Self-Insurance-cum-Protection, Journal of Risk and Uncertainty, 17:139-150.

Leif-Matthias Herborg, Christopher L. Jerde, David M. Lodge, Gregory M. Ruiz, and Hugh J. MacIsaac. 2007. Predicting Invasion Risk Using Measures Of Introduction Effort And Environmental Niche Models. Ecological Applications 17:663-674.

Horan, R.D. and E.P. Fenichel. 2007. Economics and Ecology of Managing Emerging Infectious Animal Diseases. American Journal of Agricultural Economics 89: 1232-1238.

Horan, R.D., E.P. Fenichel, K. Drury, and D.M. Lodge. 2011. Managing Ecological Thresholds in Coupled Environmental-Human Systems. Proceedings of the National Academy of Sciences of the United States of America 108: 7333-7338.

Keller, R.P. 2009. Bioeconomics of invasive species: integrating ecology, economics, policy, and management. Oxford University Press.

Kim, C.S., R.N. Lubowski, J. Lewandrowksi, and M.E. Eiswerth. 2006. Prevention or Control: Optimal Government Policies for Invasive Species Management. Agricultural and Resource Economics Review 35: 29-40.

Knowler, D. and E. Barbier. 2005. Importing Exotic Plants and the Risk of Invasion: Are Market-Based Instruments Adequate? Ecological Economics 52: 341-354.

Kobayashi, M., K. Rollins, and M.H. Taylor, 2014. Optimal Livestock Management on Sagebrush Rangeland with Ecological Thresholds, Wildfire, and Invasive Plants, Land 
Economics, 90 (4): 623-648.

Kremer, M. Integrating Behavioral Choice into Epidemiological Models of AIDS. 1996. The Quarterly Journal of Economics 111.2: 549-573.

Maler, Karl-Goran, A. Xepapadeas, and A. de Zeeuw, 2003. "The Economics of Shallow Lakes", Environmental and Resource Economics 26: 603-24

Namba, T. and S. Takahashi. 1993. Competitive Coexistence in a Seasonally Fluctuating Environment II. Multiple Stable States and Invasion Success. Theoretical Population Biology 44: 374-402.

Olson, L.J. and S. Roy. 2005. On Prevention and Control of an Uncertain Biological Invasion. Review of Agricultural Economics 27: 491-497.

Olson, L.J., and S. Roy. 2002. The Economics of Controlling a Stochastic Biological Invasion. American Journal of Agricultural Economics 84: 1311-1316.

Perrings, C. 2005. Mitigation and Adaptation Strategies for the Control of Biological Invasions. Ecological Economics 52: 315-325.

Perrings, C. and B. Walker, 1997. Biodiversity, resilience and the control of ecologicaleconomic systems: the case of fire-driven rangelands. Ecological Economics 22: 73-83.

Prasad, A. M., L. R. Iverson, M.P. Peters, J.M. Bossenbroek, S.N. Matthews, D. Sydnor, \& M.W. Schwartz. 2010. Modeling the invasive emerald ash borer risk of spread using a spatially explicit cellular model. Landscape Ecology, 25(3): 353-369.

Ranjan, R., E. Marshall, and J. Shortle. 2008. Optimal Renewable Resource Management in the Presence of Endogenous Risk of Invasion. Journal of Environmental Management 89: 273283. 
Reed, W.J. and H.E. Heras. 1992. The Conservation and Exploitation of Vulnerable Resources. Bulletin of Mathematical Biology 54: 185-207.

Rondeau, D. 2001. Along the Way Back from the Brink. Journal of Environmental Economics and Management 42: 156-182.

Shogren, J. 2000. Risk reduction strategies against the "explosive invader. The economics of biological Invasions. C. Perrings, M. Williamson, and S. Dalmazzone, eds. Edward Elgar, Cheltenham, UK, 56-69.

Shogren, J. and T.D. Crocker. 1991. Risk, Self-Protection, and Ex Ante Economic Value. Journal of Environmental Economics and Management, 20: 1-15.

Tahvonen, O. and S. Salo. 1996. Nonconvexities in Optimal Pollution Accumulation. Journal of Environmental Economics and Management, 31: 160-177.

Weitzman, M.L. 1976. On the Welfare Significance of National Product in a Dynamic Economy. Quarterly Journal of Economics 90: 156-162.

Zemel, A. 2015. Adaptation, mitigation and risk: An analytic approach. Journal of Economic Dynamics and Control 51 (2015): 133-147. 


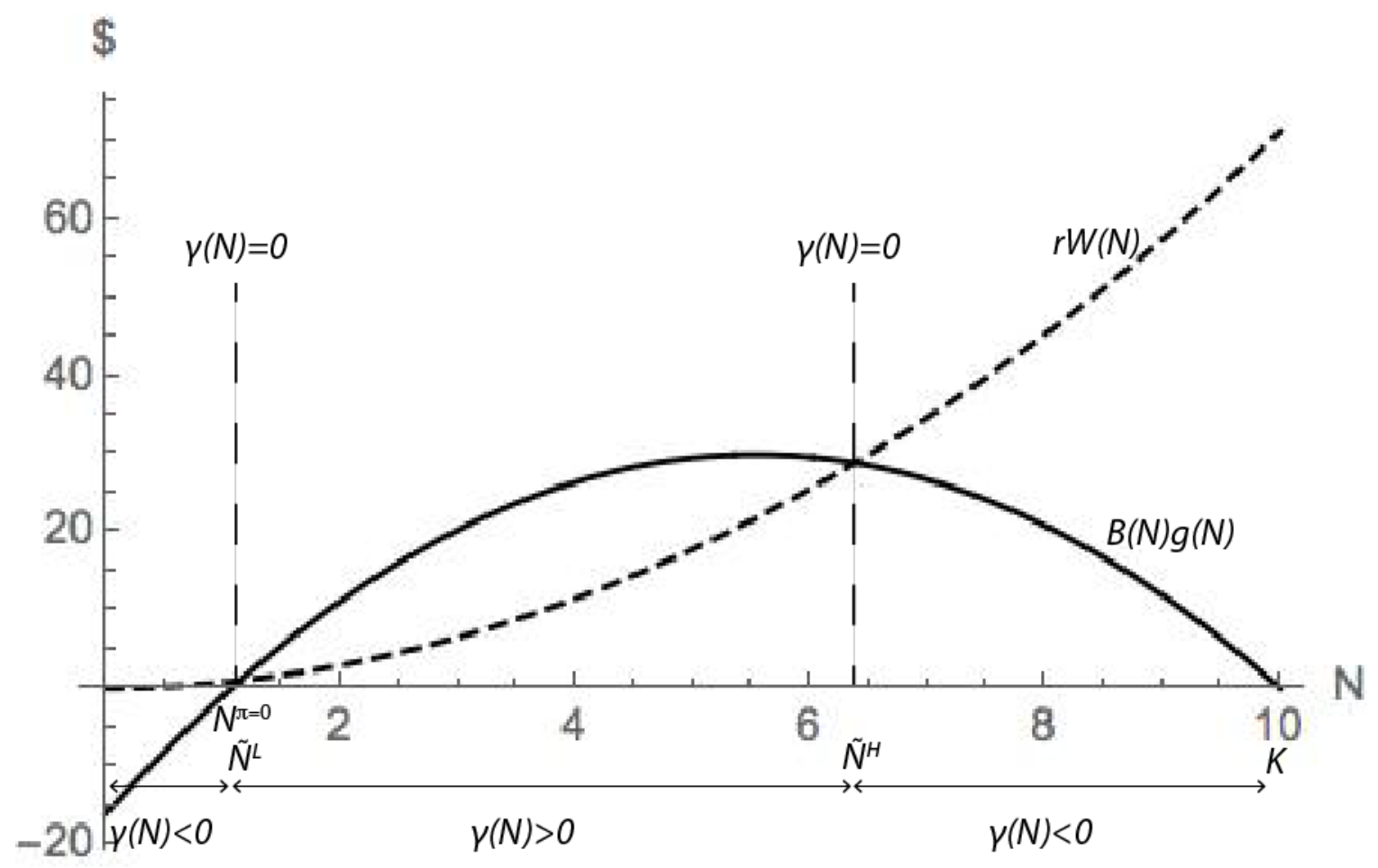

Figure 1. Characterizing $\gamma(N)$ 


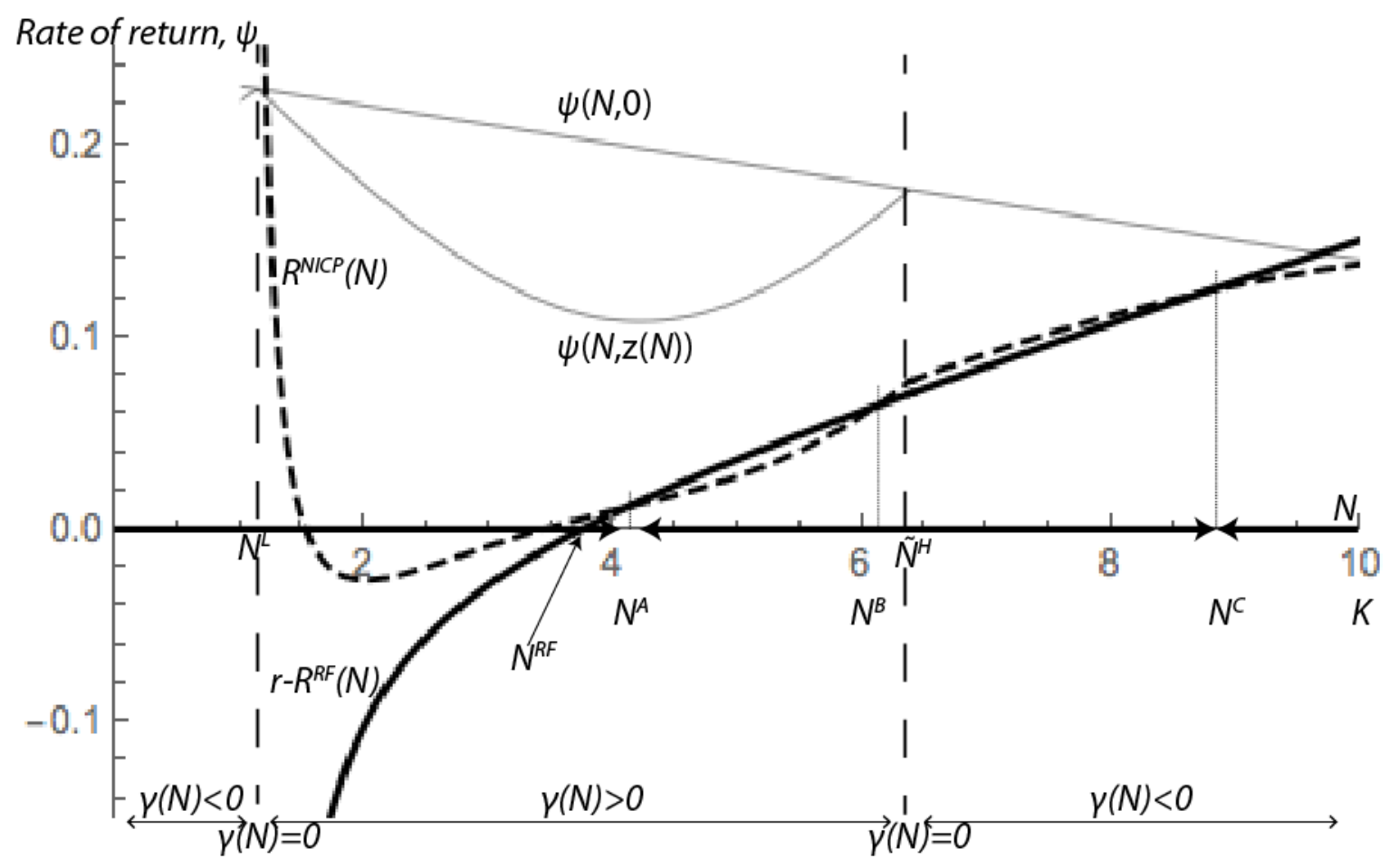

Figure 2. Multiple interior equilibria for $N$ and endogenous risk. Arrows indicate direction of movement of $N$. 


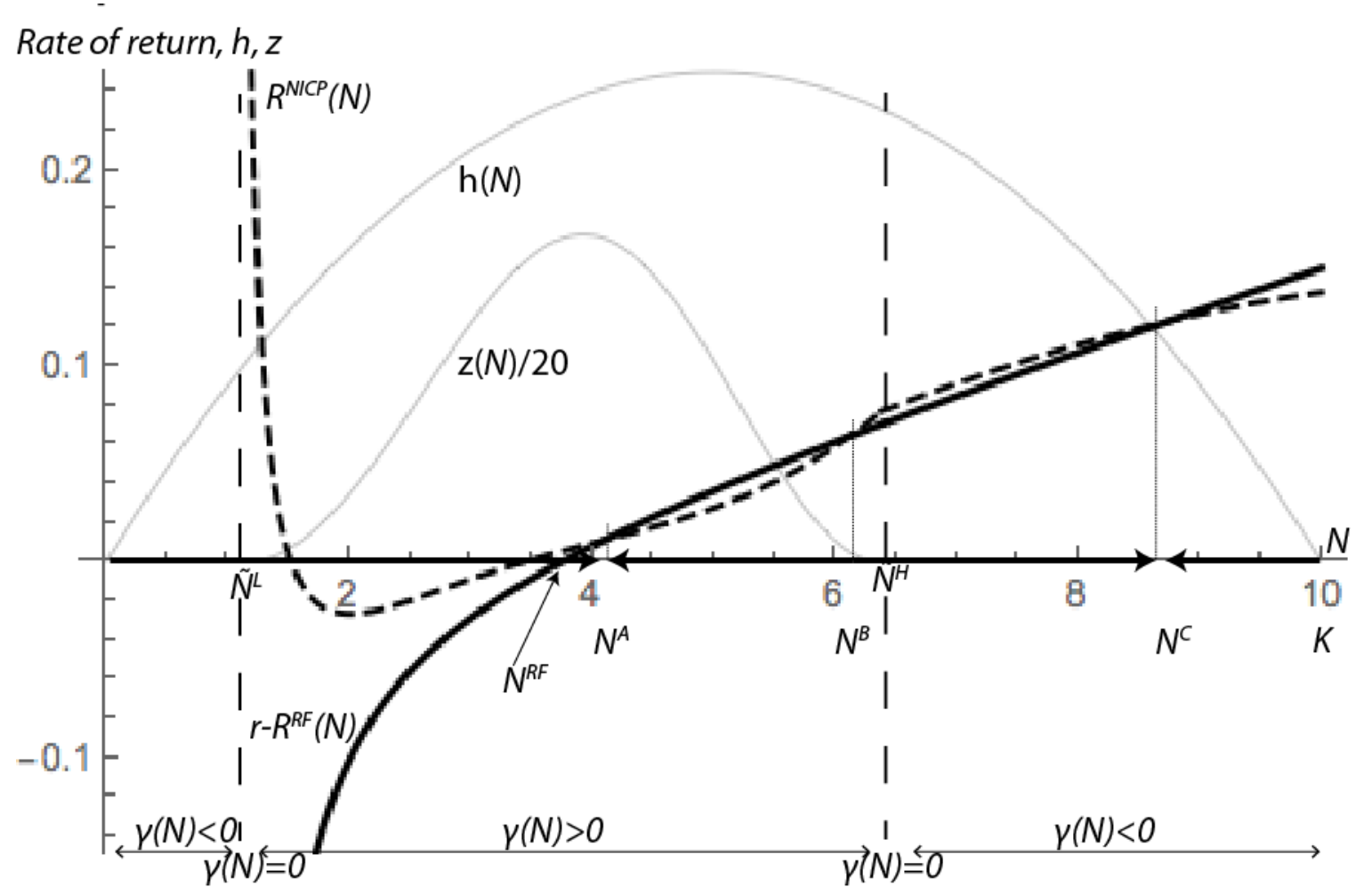

Figure 3. Multiple interior equilibria for $N$ and risk reduction. Arrows indicate direction of movement of $N$. 